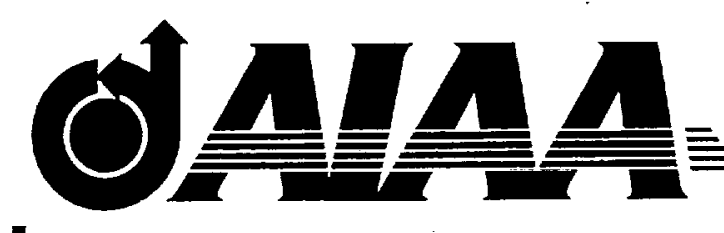

\title{
AIAA 2001-0860 \\ UNSTEADY ANALYSIS OF \\ SEPARATED AERODYNAMIC FLOWS \\ USING AN UNSTRUCTURED \\ MULTIGRID ALGORITHM
}

\section{J. Pelaez}

Department of Aerospace Engineering

Old Dominion University

Norfolk, VA

D. J. Mavriplis

ICASE

MS 132C NASA Langley Research Center

Hampton, VA

Osama Kandil

Department of Aerospace Engineering

Old Dominion University

Norfolk, VA

\section{9th AIAA Aerospace Sciences Meeting}

January 8-12 2001, Reno NV 



\title{
Unsteady Analysis of Separated Aerodynamic Flows using an Unstructured Multigrid Algorithm
}

\author{
Juan Pelaez \\ Old Dominion University \\ Hampton, Virginia \\ Dimitri Mavriplis \\ ICASE \\ NASA Langley Research Center, Hampton, Virginia \\ Osama Kandil \\ Old Dominion University \\ Hampton, Virginia
}

\begin{abstract}
An implicit method for the computation of unsteady flows on unstructured grids is presented. The resulting nonlinear system of equations is solved at each time step using an agglomeration multigrid procedure. The method allows for arbitrarily large time steps and is efficient in terms of computational effort and storage. Validation of the code using a oneequation turbulence model is performed for the well-known case of flow over a cylinder. A Detached Eddy Simulation model [1] is also implemented and its performance compared to the one equation Spalart-Allmaras ReynoldsAveraged Navier-Stokes (RANS) turbulence model [2]. Validation cases using DES and RANS include flow over a sphere and flow over a NACA 0012 wing including massive stall regimes. The project was driven by the ultimate goal of computing separated flows of aerodynamic interest, such as massive stall or flows over complex non-streamlined geometries.
\end{abstract}

\section{Introduction}

The use of time-dependent simulations for flows of practical interest is much less widespread than the use of steady-state flow simulations, due to limitations related to computational time and computational resources. However, many flows are inherently unsteady, particularly when large amounts of separation are present, and must be simulated as such in order to obtain meaningful results.

Copyright c 200I by AIAA, Inc. All Rights Reserved.
Transient flow simulations can either be based on explicit or implicit time-stepping schemes. Stability constraints limit the maximum allowable time step size of an explicit scheme, which is proportional to the smallest cell size in the entire computational mesh. Explicit schemes are well suited for unsteady applications in which the time scale of interest is comparable to the spatial scales. In these cases the mesh should be clustered only in regions of interest where it is absolutely necessary or the explicit time step can become unnecessarily small.

Explicit time steps may become too restrictive for cases characterized by larger time scales or in cases where there is a wide variation in the grid resolution. In these cases it is desirable to develop a fully implicit method in which the time step is only determined by the physics of the flow and not by the cell size. At each time step the unsteady residual must be driven to zero and this is usually done using inner iterations. The number of inner iterations needed for each time step is related to the problem being solved and the size of the physical time step used.

To develop an unsteady solver that can provide an accurate description in time of the flow is the first requirement to address before attempting to solve flows with massively separated regions that are inherently unsteady. On the other hand, it is also important to review the different techniques available to compute turbulence, which is at the core of separated flows, and will be a key parameter to determine how well the numerical scheme captures all the physical aspects of the flow. In this sense, numerical solutions of turbulent flow cases can be achieved 
using different levels of approximation. The most widespread method is to solve the Reynolds averaged Navier-Stokes equations (RANS). In the RANS equations, the turbulent fluctuations appear in the Reynolds stress term that must be modeled using any of the turbulence models available in the literature. However, a common limitation of these models is their lack of generality since the model coefficients are usually set using simple well-documented flows. In this sense, current RANS solvers are fairly successful at predicting mostly attached flows, such as a wing in cruise condition, but fail to capture a range of different off-design situations as post-stall regimes, certain high-lift configurations, and non-streamlined bodies. In general, in the cases in which the RANS approach fails, the flow is characterized by large amounts of separation in which a very wide range of scales is present in the flow. While the small scales tend to be universal in nature, the larger scales are affected by the boundary conditions. This is the main cause of the lack of generality of turbulence models, as it is difficult to model the effect of the large scales in the same way for many different types of flows.

The failure to develop a universally valid turbulence model has led to alternate approaches such as Direct Numerical Simulation (DNS) and Large Eddy Simulation (LES). DNS is the most straightforward approach to the problem. DNS consists in solving the governing equations on a mesh fine enough to capture the smallest scales contained in the flow with a scheme designed to minimize the numerical dispersion and dissipation. The drawback is extremely high cost of the DNS computation, which is proportional to at least $\mathrm{Re}^{3}$. For these reasons, DNS is generally limited to very simple flows and low Reynolds Numbers.

The flow limitations of RANS and the difficulty of using DNS for realistic applied engineering problems have generated a great interest in the Large Eddy Simulation approach (LES) for computing flows with large amounts of separation. Large Eddy Simulation is a technique between Direct Numerical Simulation and Reynolds Averaged Navier-Stokes [3]. In LES the contribution of the large scales is computed exactly and only the smallest scales in the flow are modeled. However, a major difficulty of LES, particularly for external aerodynamic flows, is that near solid surfaces all the eddies are small and the "large" and "small" eddies tend to overlap. Therefore the required grid spacing and time step gradually fall towards DNS as the solid boundary is approached [4].

Using LES to resolve near wall streaks would bring an immense penalty at industrial Reynolds numbers. In this line of reasoning the Detached Eddy Simulation (DES) approach was conceived with the idea of combining the strengths of Reynolds Averaged methods near the solid boundaries and of Large Eddy Simulation elsewhere $[1,4,5]$.

The ultimate goal of this work is to develop a large eddy simulation capability based on an existing unstructured grid Navier-Stokes solver [6] to be able to perform detached eddy simulations combing RANS near the walls and LES in massive separated regions in a non zonal manner and to compare the solutions obtained using this approach to the solutions obtained using a classical RANS approach with a SpalartAllmaras one equation turbulence model [6].

\section{Unsteady Revnolds A veraged Navier- Stokes Solver}

\subsection{Steady solver description}

The Reynolds averaged Navier-Stokes equations are discretized by a finite volume technique on meshes of mixed elements, including tetrahedra, pyramids, prisms and hexahedra. In general, prismatic elements are used in the boundary layer regions, while tetrahedra are used elsewhere.

Flow variables are stored at the grid vertices and a single unifying edge-based data-structure is used to handle all elements of the grid. Convective and viscous fluxes are discretized along edges using a central difference finite volume technique with added artificial dissipation.

The non-dimensional steady conservative form of the full Navier-Stokes equations can be written as:

$$
R(w)=0
$$

where $w$ represents the solution vector of conserved variables and $R$ represents the spatial discretization operator, or residual, which vanishes at the steady state. 
An implicit solution procedure for solving equation (1) begins with the linearization of the residual about the current time step or iteration level:

$$
\frac{\partial w}{\partial \tau}+R\left(w^{n}\right)+\frac{\partial R}{\partial w} \cdot \Delta w=0
$$

which can be solved as:

$$
\Delta w=\left[\frac{I}{\Delta \tau}+\frac{\partial R}{\partial w}\right]^{-1} \cdot\left\{-R\left(w^{n}\right)\right\}
$$

where $\tau$ is a pseudo-time used to advance the solution, and $\Delta w$ represents the new correction to be applied to the solution. Rather than inverting the large Jacobian in equation (3), a reduced Jacobian which is simpler to invert is employed. In regions of isotropic grid cells, only the block diagonals of the Jacobian are retained, leading to a block-Jacobi or point-implicit scheme. In the boundary layer regions, where high grid stretching is present, lines are constructed in the direction normal to the solid wall boundary and the Jacobians along these lines are inverted, using a block tridiagonal solution algorithm. This procedure relieves the stiffness associated with high grid stretching in these regions, thus providing more rapid convergence [7].

This locally implicit scheme is used as the smoother on all levels of an agglomeration multigrid algorithm. The multigrid algorithm constructs coarse level grids automatically by fusing together neighboring fine grid control volumes to form a smaller number of large coarse grid control volumes. Convergence acceleration is achieved by cycling back and forth between the fine and coarse grid levels of the multigrid sequence, using the locally implicit solution procedure as a solver on each grid level [7].

The unstructured multigrid solver is parallelized by partitioning the domain using a standard graph partitioner [8] and communicating between the various grid partitions running on individual processors using the MPI messagepassing library [9]. The solver can be run on distributed or shared memory machines, including clusters of personal computers.

\subsection{Unsteady term implementation}

The unsteady form of the governing equations is obtained by adding the time derivative to equation (1):

$$
\frac{\partial w}{\partial t}+R(w)=0
$$

where $R(w)$ denotes the discretization of the spatial derivative terms, as previously.

Making use of a second-order accurate three point backward approximation for the time derivative and evaluating $R(w)$ at time level $(\mathrm{n}+1)$, we obtain $[10,11,12]$ :

$$
\frac{3}{2 \cdot \Delta t} \cdot w^{n+1}-\frac{2}{\Delta} \cdot w^{n}+\frac{1}{2 \cdot \Delta t} \cdot w^{n-1}+R\left(w^{n+1}\right)=0
$$

We may now define and unsteady residual as:

$$
R^{*}(w)=\frac{3}{2 \cdot \Delta t}+R(w)-S\left(w^{n}, w^{n-1}\right)
$$

with

$$
S\left(w^{n}, w^{n-1}\right)=\frac{2}{\Delta t} \cdot w^{n}-\frac{1}{2 \cdot \Delta t} \cdot w^{n-1}
$$

where $w^{n+1}$ is the approximation to $w$ and the source term $S\left(w^{n}, w^{n-1}\right)$ remains fixed throughout the solution procedure at each time step. In this form, the non-linear problem to be solved at each time-step reduces to $\mathrm{R}^{*}(\mathrm{w})=0$. Using this redefinition of the residual, the same iterative multigrid procedure employed to solve the steady-state problem can be used to solve the non-linear unsteady residual at each physical time step $[10,11,12]$.

The solution procedure consists of an outer loop over the physical time steps, used to advance the problem in time, and an inner (multigrid) loop over pseudo-time used to drive the unsteady residual to zero at each time level. While the maximum size of the physical time step is only determined by the physics of the problem, the convergence of the inner sub-iterations for each time step varies inversely with the size of the physical time step.

\section{Unsteady solver validation}

The flow around a circular cylinder is a wellknown case, which has been widely studied computationally and experimentally. This case is used as the basis for validation of the unsteady RANS solver, and for assessing grid resolution 
and time step requirements for accurately predicting the vortex shedding frequency observed in the cylinder flow. Two different meshes of 252,000 and 631,000 grid points and three different time steps of $0.5,0.25$ and 0.1 were used. The time is non-dimensionalized as $t$ $=t_{0} /\left(d / U_{\infty}\right)$ where $d$ is diameter of the cylinder and $U_{\infty}$ is the freestream velocity.

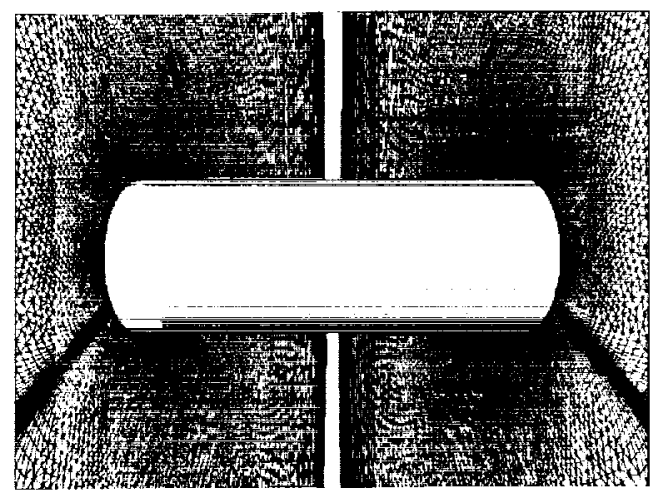

Figure 1: $3 D$ View of cylinder and lateral walls

The one equation Spalart-Allmaras turbulence model [2] was used for all calculations in fully turbulent mode. In all cases the agglomeration multigrid strategy was used with four levels. The Mach number is 0.2 and the Reynolds number is 1200 for this case. All runs were performed in parallel using 16 processors of a Pentium PC cluster at ICASE.

The computational domain in the plane normal to the cylinder span has an aspect ratio of 1 and $a$ side length of 100 cylinder diameters. A span of two cylinder diameters is employed, and inviscid (slip velocity) boundary conditions are applied at the end-walls. The three-dimensional simulations reported herein were also compared with two-dimensional simulations of flow around a circle using a validated twodimensional unstructured solver [12], and found to agree well in terms of force coefficient histories and shedding frequency.

Table 1 shows the Strouhal Numbers computed for each mesh and each time step of the threedimensional simulations. Convergence is achieved as the time step is reduced and the mesh size increased. A second-order accuracte convergence behavior is observed as the timestep is reduced, validating the accuracy of the three-point backwards difference scheme used to discretize the time step. From the smallest time step results, the solution can be seen to be grid converged, at least with respect to the prediction of the vortex shedding frequency. The computed Strouhal number compares very well to the experimental value of $\mathrm{St}=0.21$ given by Dresher $[13,14]$. Figure 2 shows the time history of the lift coefficient, while the oscillatory pattern corresponding to the vortex shedding is shown in Figure 3.

\begin{tabular}{|c|c|c|c|}
\cline { 3 - 4 } \multicolumn{1}{c|}{} & \multicolumn{3}{|c|}{ Time Step } \\
\cline { 3 - 5 } \multicolumn{1}{c|}{} & $\mathbf{0 . 5}$ & $\mathbf{0 . 2 5}$ & $\mathbf{0 . I}$ \\
\hline $\begin{array}{c}\mathbf{0 . 2 5 2} \\
\text { Million }\end{array}$ & 0.19249 & 0.20304 & 0.20833 \\
\hline $\begin{array}{c}\mathbf{0 . 6 3 1} \\
\text { Million }\end{array}$ & 0.19379 & 0.20408 & 0.20833 \\
\hline
\end{tabular}

Table 1. Predicted Strouhal Number for Various Grid and Time Step Sizes

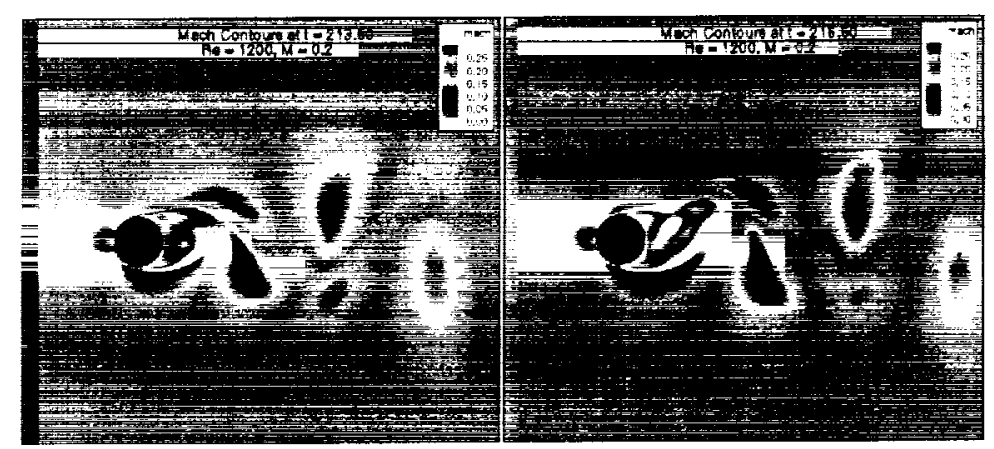

Figure 2: Mach contours at two different time states for flow over circular cylinder. Mach $=$ $0.2, \operatorname{Re}=1200$. 

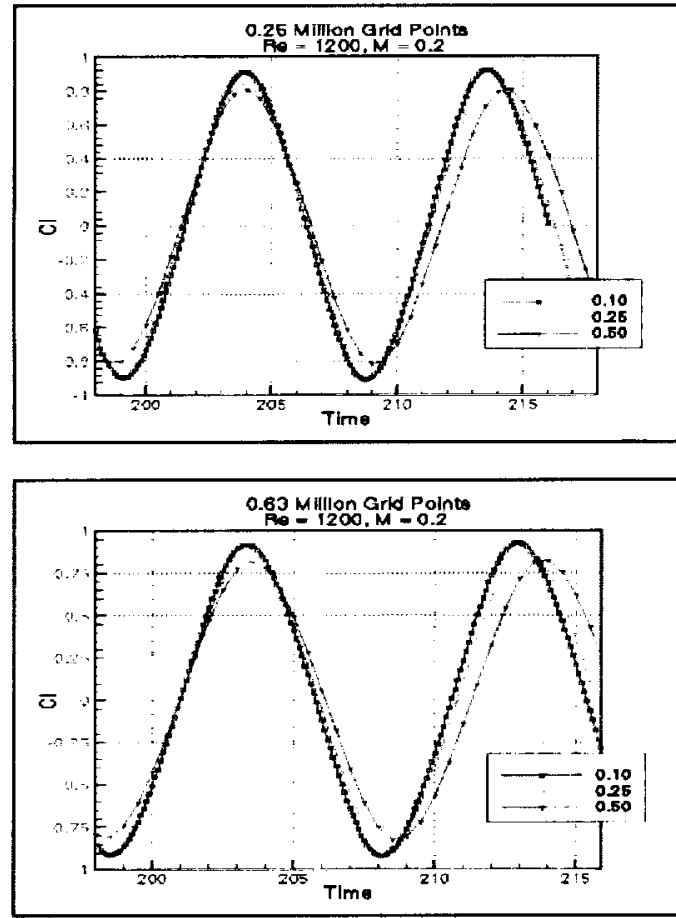

Figure 3. Computed LiftCoefficient Time History for Flow over Circular Cylinder using three different time steps. Mach $=0.2, \operatorname{Re}=1200$

Mean pressure coefficient distribution over the cylinder. $M=0.2, R \theta=1200$

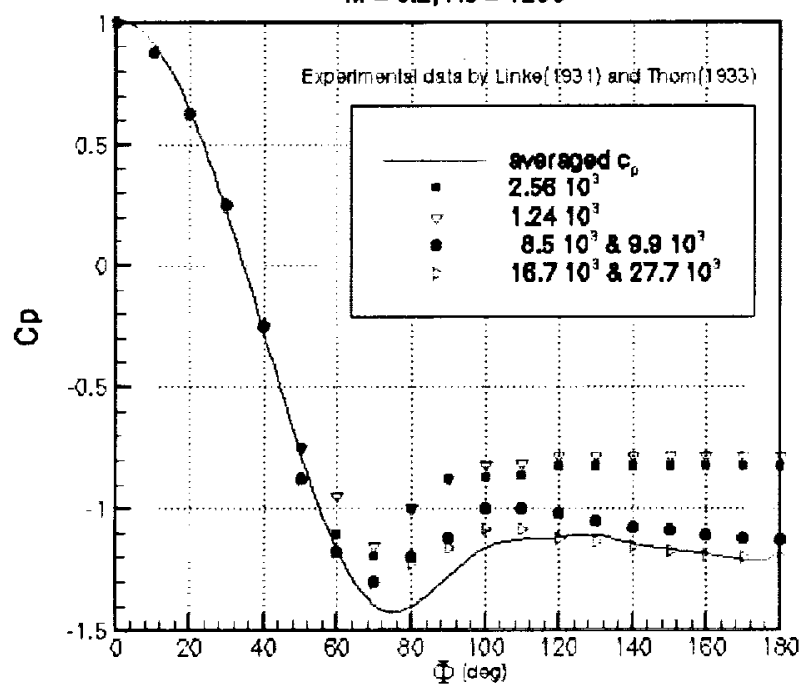

Figure 4. Mean Surface Pressure Coefficient Distribution for Flow over Circular Cylinder at Mach $=0.2, \operatorname{Re}=1200$, Compared with Experimental Data at Various Reynolds Numbers
Figure 4 shows the pressure distribution over the surface of the cylinder. The pressure distribution was computed by averaging results at different times distributed along several oscillations. For this calculation the finest mesh and the smallest time step was used. As can be observed from Figure 4 , the computed pressure distribution compares closely to experimental results at a higher Reynolds number than the one used for these computations. This is likely due to the use of the turbulence model in fully turbulent mode, in order to avoid the issues of transition prediction. Similarly, the backpressure obtained of $\mathrm{C}_{\mathrm{pb}}=-1.20$ compares closely to the $\mathrm{C}_{\mathrm{pb}}=$ 1.20 at $\operatorname{Re}=27,700$ measured by Linke $[13,15]$. The mean value of the drag coefficient computed is $C_{d}=1.3$ compared to $C_{d}=1.2$ as measured by Wieselsberger $[13,16]$ for $\mathrm{Re}=30,000$.

\section{Detached Eddy Simulation.}

Detached Eddy Simulation (DES) is a hybrid technique that combines RANS and LES in a non-zonal manner. DES is based on the SpalartAllmaras one equation RANS model [2] in which the length scale $d$, which is traditionally taken as the nearest distance at any given point to the closest wall, is replaced as the minimum between the distance to the wall and a length proportional to the local grid spacing:

$\mathrm{d}_{\mathrm{DES}}=\min \left(\mathrm{d}, \mathrm{C}_{\mathrm{DES}} \Delta \mathrm{x}\right)$

where $C_{D E S}$ represents a model constant which has been taken as 0.65 in previous work $[1,17]$. Traditionally, on structured grids $[1,17,18], \Delta x$ is taken as the maximum grid spacing over all three directions. In our particular case, the definition of $\Delta \mathrm{x}$ has been modified for unstructured grids by taking it as the maximum edge length touching a given vertex. In boundary layer regions, $\Delta x$ far exceeds the distance to the wall $\mathrm{d}$, and the standard Spalart-Allmaras RANS turbulence model is recovered. However, away from the boundaries $\mathrm{d}$ exceeds $\mathrm{C}_{\mathrm{DES}} \Delta \mathrm{x}$ and the models turns into a simple one equation subgrid-scale (SGS) model with the mixing length proportional to the grid spacing. This effect is illustrated by plotting contours of the distance or length scale function for both the RANS and DES models in Figure 5, where it is observed that both models employ the same length scales 
near the wall, but use vastly differing length scales in the regions far removed from the wall, where the DES model reverts to an LES mode and a Smagorisnky-like expression for the eddy viscosity is obtained.
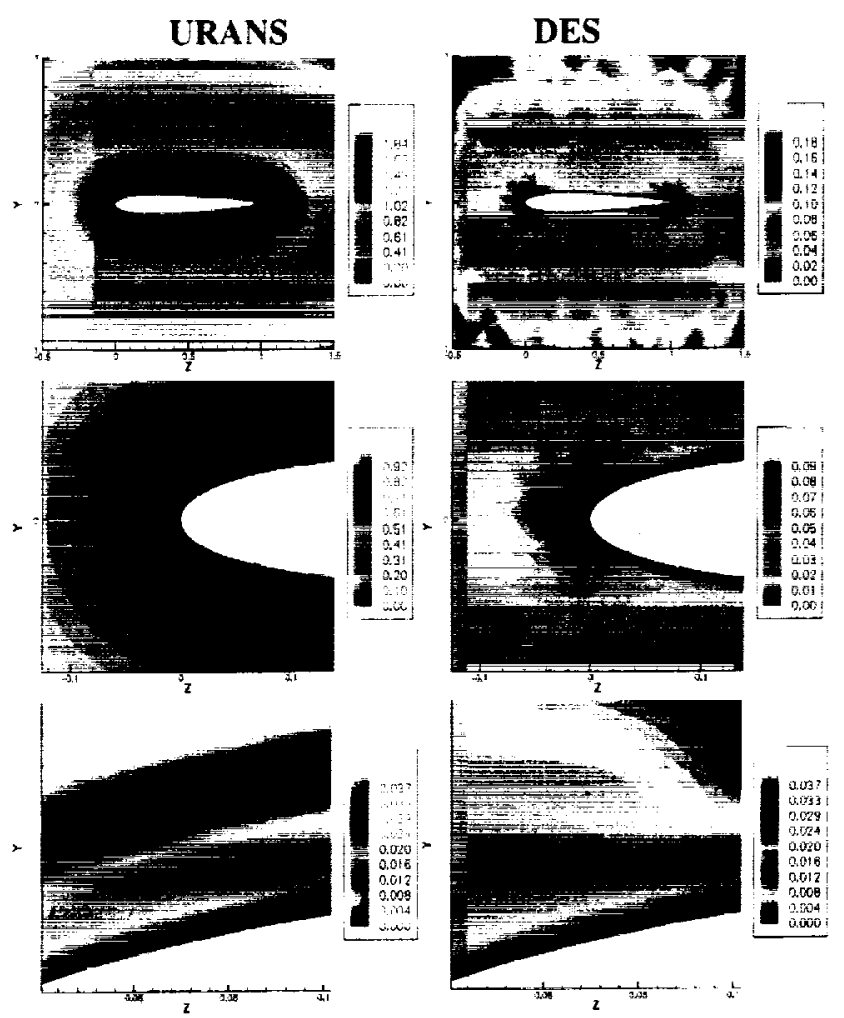

Figure 5. Distance function/Length Scale comparison between URANS and DES.

\section{Validation.}

\subsection{Flow around a Sphere.}

DES is applied to predict the flow around a sphere. Similarly to the flow around a circular cylinder, this case has been widely studied using experimental and computational approaches. It has been shown that over a wide range of Reynolds numbers $\left(280<\operatorname{Re}<3.7 \times 10^{5}\right)$ the flow is characterized by the vortex shedding with large-scale vorticity emanating from the shear layer which separates from the surface of the sphere. In our case a Reynolds number of $10^{4}$ is used, corresponding to the sub-critical regime (laminar boundary layer separation). This is similar to the computations performed by
Constantinescu [17], which provides a comparative basis for our results.

The main goal of this case is to compare the results obtained using traditional URANS and DES. The comparison includes the time history of integral parameters such as drag coefficient and mean distribution of pressure around the sphere.

\subsubsection{Numerical approach.}

The flow over a sphere is calculated for a Reynolds number of $10^{4}$ and a Mach number of 0.2 on an unstructured mesh of 767,000 vertices. The computational domain is a cube of 100 sphere diameter lengths in each direction. This large domain is chosen to ensure that the downstream boundary condition does not lead to spurious oscillations within the domain. Near the surface of the sphere, the normal grid spacing is $10^{-4} \times \mathrm{d}$, where $\mathrm{d}$ represents the diameter of the sphere.

For the unsteady calculations two different time steps of $0.1 \times \mathrm{d} / \mathrm{U}_{\infty}$ and $0.05 \times \mathrm{d} / \mathrm{U}_{\infty}$ were used. In all cases the multigrid strategy was used with four grid levels. All runs were performed on 32 processors of a Pentium PC cluster at ICASE.

For the DES runs, the $C_{D E S}$ value used was 0.65 . This value was chosen based on previous studies by Shur et al. [1], where the value was calibrated in isotropic turbulence. Moreover, Constantinescu et al. [17] prove this value of $\mathrm{C}_{\mathrm{DES}}$ as the most appropriate for their calculations of the flow over a sphere at $\mathrm{Re}=$ $10^{4}$. A parallel study is being conducted to examine the effects of variations in the value of $\mathrm{C}_{\mathrm{DLES}}$ using the same test case of decaying homogeneous turbulence in a box as done by Shur et al. [1].

\subsubsection{Code validation.}

Following the procedure used by Constantinescu et al. [17], the accuracy of the numerical approach was established by comparing the results obtained in the steady regime at Reynolds number 250 with previous computational and experimental results.

The drag coefficient is compared in Table 2 to other simulation results and experimental data. Because previous results were based on 
incompressible simulations, and the current solver is a density based compressible formulation, the importance of compressible effects was also investigated by running the simulation at Mach numbers of 0.2 and 0.1 , both with and without a low Mach number preconditioner $[18,19]$. The agreement is satisfactory for all the cases tested.

\begin{tabular}{|c|c|}
\cline { 2 - 2 } \multicolumn{1}{c|}{} & $\mathbf{C d}$ \\
\hline $\begin{array}{c}\text { Constantinescu et } \\
\text { al. } \\
(\mathbf{2 0 0 0})\end{array}$ & 0.70 \\
\hline $\begin{array}{c}\text { Johnson and Patel } \\
(\mathbf{1 9 9 9 )}\end{array}$ & 0.70 \\
\hline Experimental & $0.70-0.72$ \\
\hline $\mathbf{M}=\mathbf{0 . 1}$ & 0.7141 \\
\hline $\mathbf{M}=\mathbf{0 . 2}$ & 0.7015 \\
\hline $\begin{array}{c}\mathbf{M}=\mathbf{0 . 1} \\
\text { Low Mach } \\
\text { Number pre- } \\
\text { conditioner. }\end{array}$ & 0.6961 \\
\hline $\begin{array}{c}\mathbf{M}=\mathbf{0 . 2} \\
\text { Low Mach } \\
\text { Number pre- } \\
\text { conditioner. }\end{array}$ & 0.6950 \\
\hline
\end{tabular}

Table 2. Computed Steady Drag Coefficient for Flow over Sphere at $R e=250$ compared with Experimental and Previous Computational Values

\subsubsection{Unsteady Results.}

For the unsteady runs, the flow around a sphere is computed at a Mach number of 0.2 , without any additional low Mach number preconditioning, and a Reynolds number of $10^{4}$. At this Reynolds number the detached vortex sheet from the sphere is fully turbulent while the boundary layer on the sphere remains laminar. The Strouhal number associated with the vortex shedding at this Reynolds number is in the range of 0.185-0.200 depending on the investigation. The large scatter of the data is mainly due to the influencing parameters and the measuring methods of the different investigations.

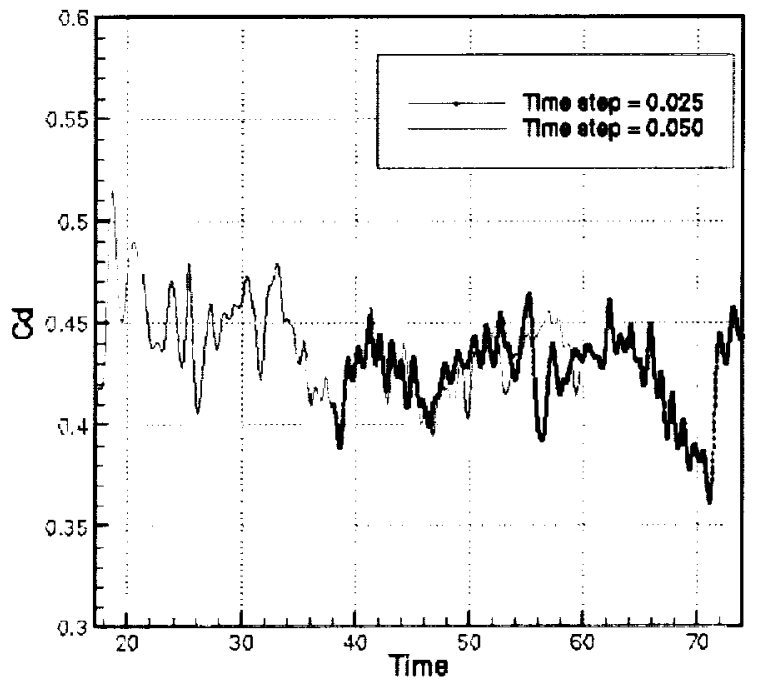

Figure 6. DES Time history of Drag Coefficient for flow over Sphere at Mach $=0.2, R e=10,000$ using two different time steps

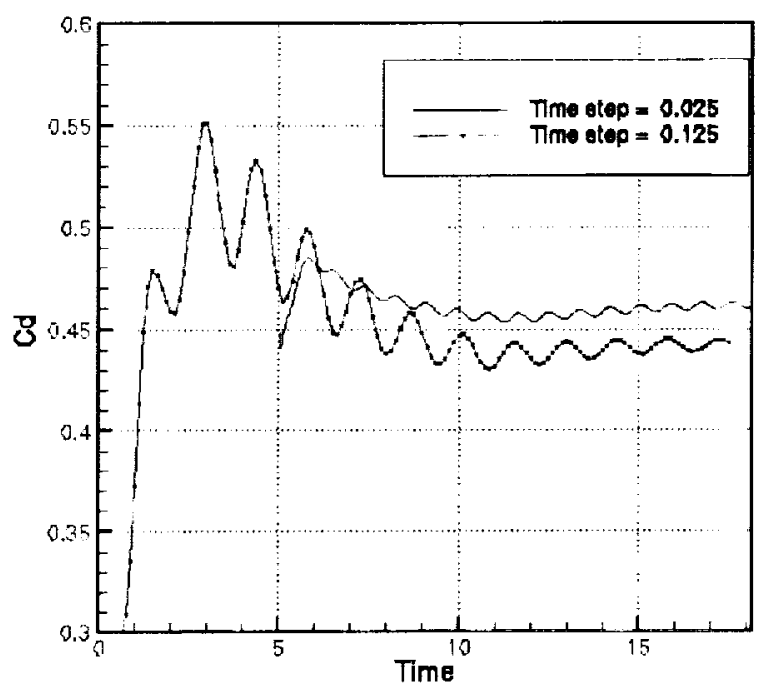

Figure 7. URANS Time history of the drag coefficient for flow over Sphere at Mach $=0.2$, $R e=10,000$ using two different time steps 


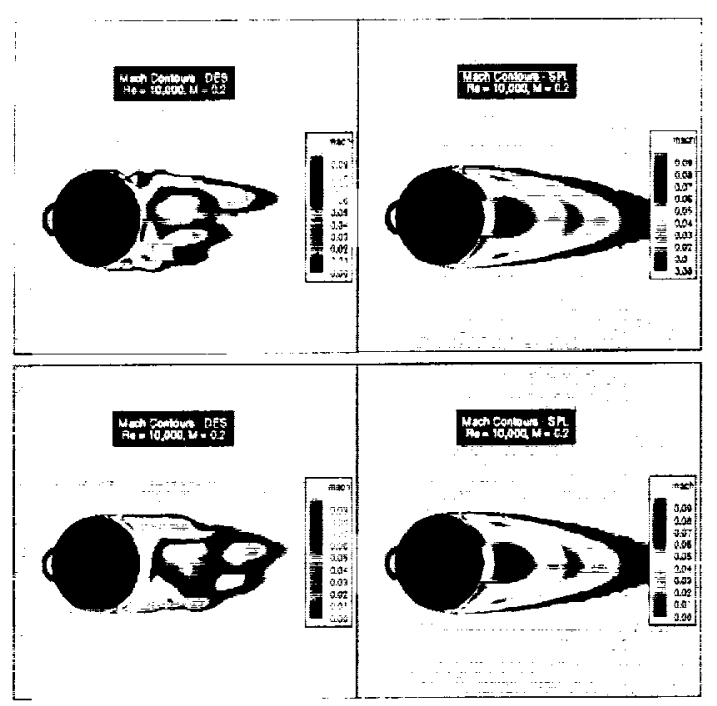

Figure 8. Comparison of URANS and DES Mach Contours for flow over sphere.

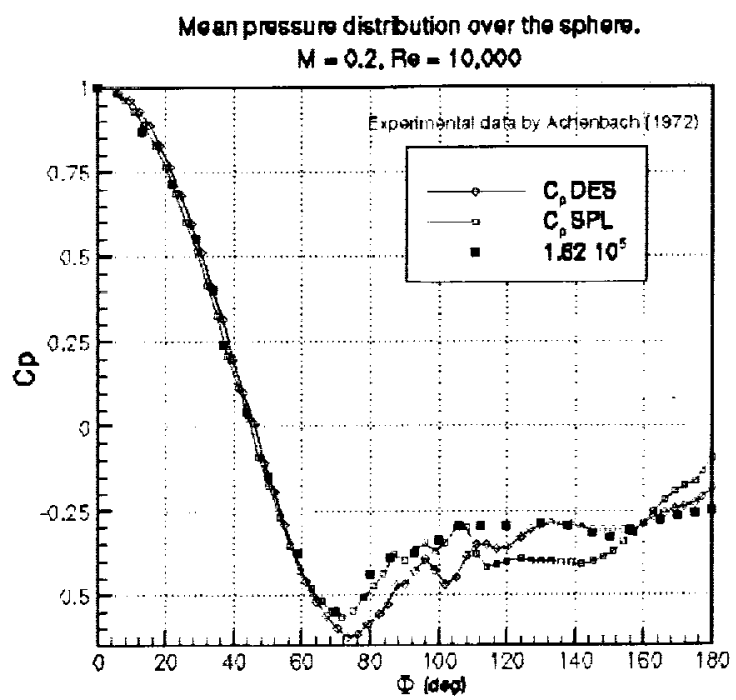

Figure 9.. URANS and DES Average Surface

Pressure Distributions Compared with

Experimental Data at Various Reynolds

Numbers for flow over Sphere

Mach $=0.2 . \operatorname{Re}=10,000 \mathrm{~s}$

The time history of the drag coefficient is shown in Figures 6 and 7 and reveals important differences between URANS and DES. The mean value of the drag coefficient in both cases is close to the experimentally reported value of 0.40 from Schlicting [20]. However, the frequency content in each case is completely different. The URANS simulation appears to damp out most of the oscillations present in the DES run, while the DES runs show a very chaotic oscillatory pattern quite similar to the solutions obtained by Constantinescu et al [17]. Spectral analysis of the time-dependent drag coefficient history reveals a peak corresponding to a Strouhal number of 0.1 which is not in agreement with the values 0.18 to 0.2 reported experimentally [21]. This may be due to an insufficiently long time history sample, since less than three full periods of this frequency are present in our sample.

The Mach number contours depicted in Figure 8 corroborate the difference in the predicted flow using regular Spalart-Allmaras RANS turbulence model and detached eddy simulation (DES). DES shows a wider range of scales present in the flow while regular RANS models tend to smooth out the smaller scales. This is the effect expected from DES since the length scale redefinition increases the magnitude of the destruction term in the Spalart-Allmaras model, drawing down the eddy viscosity and allowing instabilities to develop. Predictions of the mean pressure distribution over the surface of the sphere are shown in Figure 9. The surface pressure is seen to match well with experimental results at $\mathrm{Re}=165,000$ reported by Achenbach [21], which is in agreement with the results reported by Constantinescu [17]. The average computed separation angle of $81^{\circ}$ compares resonably to experimental vale of $82.5^{\circ}$.

\subsection{Flow over a NACA0012 airfoil.}

RANS and DES were used to compute the flow over a NACA0012 airfoil at a Reynolds number of $10^{5}$ and a Mach number of 025 . Runs were performed for 10 different angles of attack ranging from 0 to 16 . Our main interest was to study and compare RANS and DES in the stall regime. Other studies (Shur et al. [1]) have focused their interest in higher angles of attack, such as 45,60 or even 90 degrees, showing very good and promising results in which DES is within $10 \%$ of the experimental values, while traditional RANS predict results with $40 \%$ errors. All runs are computed as fully turbulent to avoid having to trigger the transition point. A mesh of 966,00 node points is used with a first grid spacing normal to the solid boundary of $10^{-5}$ chords. The spanwise boundary conditions are slip (inviscid) walls, and the span of the geometry is two chords lengths.

In this case, due to time and computing constraints, only one time step of $0.25 \times \mathrm{c} / \mathrm{U}_{\infty}$ 
was used, where $c$ is the airfoil chord. The calculations were carried out for a maximum of 50 time units for the post-stall cases, where a time unit represents the time it takes for the undisturbed far-field flow to travel one chord length.

\subsubsection{Results.}

The time history of the lift and drag coefficient show good agreement between URANS and DES for angles of attack bellow 11.5 degrees. This was expected since for pre-stall conditions the DES model operates primarily in the RANS mode. However, for the post-stall condition, i.e. angles of attack over 11.5, the time history of the force coefficients obtained using URANS and DES showed differences similar to the ones observed for the sphere case. The time history of the DES results shows higher unsteadiness that URANS, indicating that more scales are being captured in the separated region. The DES simulation predicts a more severe stall than the URANS results, i.e. lower post-stall lift and higher drag. However, both computations stall at the same incidence and comparison with experimental data [22] is not sufficiently close to favor agreement with one method over the other. This is in contrast to previous work [1] where DES was reported to provide closer agreement with experiement for very high angles of attack, such as 45 to 90 degrees. However, little attention was devoted to the prediction of the onset of stall in [1].

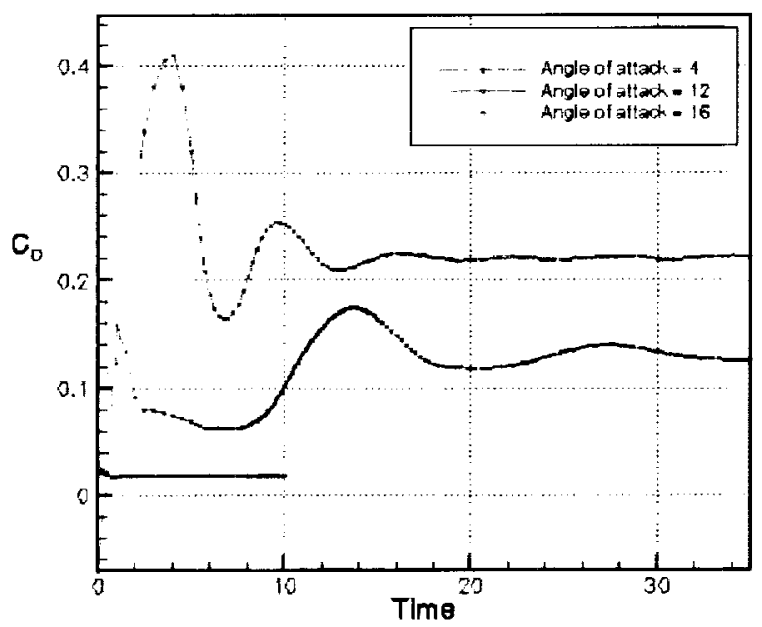

Figure 10: Sample URANS Drag Coefficient Time History for Flow over NACA0012 Wing at Various Angles of Attack

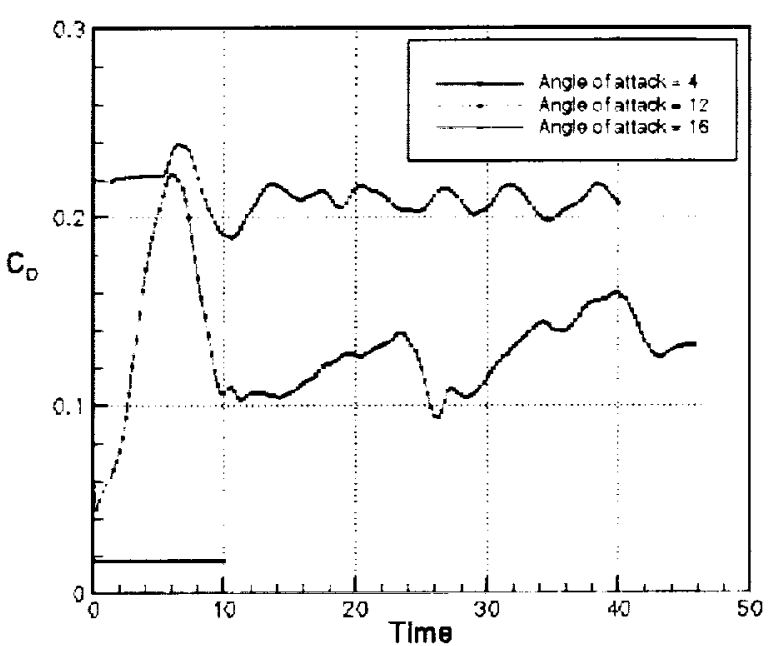

Figure 11. Sample DES Drag Coefficient Time History for Flow Over NACAOOI2 Wing at Various Angles of Attack

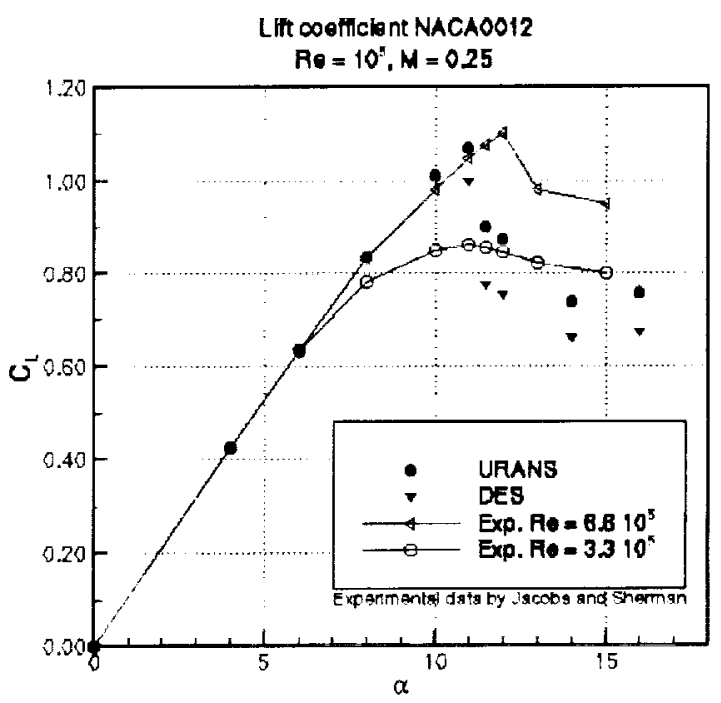

Figure 12. Comparison of Computed Lift Coefficient versus Angle of Attack for URANS and DES versus Experimental Data [22] at two Different Reynolds Numbers 


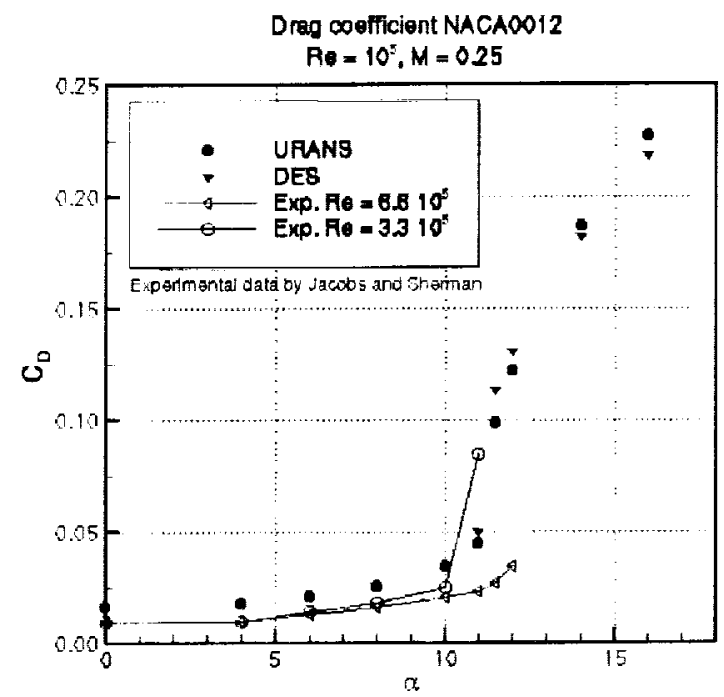

Figure 13.. Comparison of Computed Drag Coefficient versus Angle of Attack for URANS and DES versus Experimental Data [22] at two Different Reynolds Numbers

\section{Conclusions.}

A steady-state unstructured multigrid solver was extended to unsteady flows and validated by computing the flow over a circular cylinder. Results show good agreement with experimental results in Strouhal number, pressure distribution , and flow patterns over the cylinder.

A Detached Eddy Simulation (DES) technique was implemented in the code and compared with regular URANS by computing the flow over a sphere and the flow over a NACA0012 airfoil. The results for the sphere show that DES provides a better description of the flow in the separated regions. This was concluded from the time history of the force coeffcients and from the pressure distribution over the surface of the sphere. The time history of the drag coefficient computed using DES showed small scales present in the flow that URANS did not capture. The Strouhal number obtained does not match the experimental value but longer time histories are required for a more definite determination of this values.
Prediction of the onset of stall for a NACA0012 wing was not substantially improved using the DES approach over the URANS approach. However, DES has been reported to provide better agreement with experiement at very high angles of attack, and this will be investigated in future work. Additional work is also underway to simulate the decay of homogeneous isotropic turbulence in a periodic domain, in order to calibrate the DES model for unstructured grids.

\section{References.}

1. Shur M., Spalart P. R., Strelets M., Travin A. "Detached-eddy Simulation of an Airfoil at High Angle of Attack", 4 th Int. Symp. Eng. Turb. Modeling and Measurements, May 24- 26, 1999, Corsica.

2. Spalart, P. R. and Allmaras S. R., "A one equation turbulence model for aerodynamic flows", La Recherche Aerospatiale, 1, pp 521., 1994

3. Piomelli, U. "Large -eddy Simulation of Turbulent Flows"' TAN Report No. 767. UILU-ENG-94-6023, September 1994.

4. Spalart, P. R., Jou, W. H., Strelets, M. and Allmaras, S. R., "Comments on the Feasibility of LES Wings and on Hybrid RANS/LES Approach",First AFOSR InternationalConference on DNS/LES, Rouston, Louisiana, USA, 1997.

5. Shur, M., Spalart P. R., Strelets, M., and Travin, A. K., "Navier-Stokes simulation of shedding turbulent flow past a circular cylinder with a backward splitter plate" $4^{\text {th }}$ Int. Symp. Eng. Turb. Modeling and Measurements, May 24- 26,1999,Corsica .

6. Mavriplis, D.J., , "Viscous Fow Analysis Using a Parallel Unstructured Multigrid Solver", AIAA Journal, Vol 38, No. 11 pp.2067-2076, Nov 2000.

7. Mavriplis, D. J. “Directional Agglomeration Multigrid Techniques for High-Reynolds Number Viscous Flows", AIAA paper 980612, Jan 1998. 
8. Karypis, G. and Kumar, V., " A Fast and High Quality Multilevel Scheme for Partitioning Irregular Graphs", University of Minesota Tech Report 95-035, 1995.

9. Gropp, W., Lusk, E., and Skjellum, A., "Using MPI: Portable Programming with Message Passing Interface', MIT Press, Cambridge, MA, 1994.

10. Jameson, A., “Time Dependent Calculations using Multigrid with Applications to Unsteady Flows past Airfoils and Wings",AIAA paper 91-1596, July 1991.

11. Melson, N. D., Sanetrick, M. D., and Atkins, H. L., "Time-Accurate Navier-Stokes Calculations using Multigrid Acceleration", in Proc. of $6^{\text {th }}$ Copper Mountain Multigrid Conference, pp. 423-439, 1993.

12. Venkatakrishnan, V., Mavriplis, D. J., "Computation of Unsteady Flows over Complex Geometries in Relative Motion", Proc. of the $1^{\text {st }}$ AFOSR Conf. On Dynamic Motion CFD, Eds. L. Sakell, D. Knight,pp 95-112, June 1996.

13. Zdravkovich, M. M., "Flow Around Circular Cylinders : Vol 1: Fundamentals", Oxford Science Publications, Oxford UK, 1997.

14. Dresher, H., 'Measurements of TimeVarying Pressure on a Cylinder in CrossFlow", Zeitschrift fur Flugwissenschaften, 4, pp 17-21, 1956.

15. Linke, W. "New Measurements on Aerodynamics of Cylinders Particularly Their Friction Resistance", Physikalische Zeitschrift, 32, 900-14,1931.

16. Wieyselsberger, "New Data on the Law of Hydro- and Aerodynamic Resistance", Physikalische Zeitschrift, 22, 321-8, 1921.

17. Constantinescu G. S., Squires K. D.,"LES and DES Investigations of Turbulent Flow over a Sphere", AIAA paper 2000-0540, Jan 2000.

18. Weiss, J. M., Smith, W. A., "Preconditioning Applied to Variable and
Constant Density Time-Accurate Flows on Unstructured Meshes", AIAA paper 942209, June 1994.

19. Turkel, E., "Preconditioning Squared Methods for Multidimensional Aerodynamics", AIAA paper 97-2025-CP, June 1997.

20. Schlicting, H., "Boundary Layer Theory", Seventh Edition, McGraw Hill, New York, NY, 1979.

21. Achenbach, E., 'Experiments on the Flow Past Spheres at Very High Reynolds Numbers", J. Fluid Mech., 54(3), pp.565$575,1972$.

22. Jacobs, E. N., and Sherman, A., " Airfoil Section Characteristics as Affected by Variations of the Reynolds Number", NACA Technical Report 586, 1937. 
